\title{
Anti-inflammatory effects of flavonoids and phenylethanoid glycosides from Hosta plantaginea flowers in LPS-stimulated RAW 264.7 macrophages through inhibition of the NF-KB signaling pathway
}

Li Yang ${ }^{1}$ and Junwei He $2^{*}$

\begin{abstract}
Background: The flower of Hosta plantaginea (Lam.) Aschers has traditionally been used in China as an important Mongolian medicine for the treatment of inflammatory diseases with limited scientific evidence. In previous studies, 16 flavonoids and 3 phenylethanoid glycosides (1-19) were isolated from the ethanolic extract of H. plantaginea flowers. Nevertheless, the anti-inflammatory effects of these constituents remain unclear. In the present study, the anti-inflammatory effects of these 19 constituents and their underlying mechanisms were assessed in lipopolysaccharide (LPS)-stimulated RAW 264.7 macrophages.

Methods: The viability of RAW 264.7 macrophages was detected by Cell Counting Kit-8 (CCK-8) assay. Meanwhile, nitric oxide (NO) production was measured by Griess assay, while the secretion of tumor necrosis factor a (TNF-a), prostaglandin E2 (PGE2), interleukin $1 \beta$ (IL-1 $\beta$ ) and IL-6 in LPS-induced macrophages was determined by enzymelinked immunosorbent assay (ELISA). Furthermore, the protein expression of nuclear factor kappa B (NF-kB) p65 and phosphorylated NF-kB p65 was evaluated by Western blot analysis.
\end{abstract}

Results: All constituents effectively suppressed excessive NO production at a concentration of $40 \mu \mathrm{M}$ with no toxicity to LPS-induced RAW 264.7 macrophages. Among them, five flavonoids (1, 4-6 and 15) and one phenylethanoid glycoside (17) remarkably prevented the overproduction of $\mathrm{NO}$ with median inhibitory concentration $\left(\mathrm{IC}_{50}\right)$ values in the range of 12.20-19.91 $\mu \mathrm{M}$. Moreover, compounds 1, 4-6, 15 and $\mathbf{1 7}$ potently inhibited the secretion of TNF-a, PGE2, $\mathrm{IL}-1 \beta$ and $\mathrm{IL}-6$, and had a prominent inhibitory effect on the down-regulation of the phosphorylated protein level of NF-KB p65.

Conclusion: Taken together, compounds 1, 4-6, 15 and $\mathbf{1 7}$ may be useful in managing inflammatory diseases by blocking the NF-KB signaling pathway and suppressing the overproduction of inflammatory mediators.

Keywords: Hosta plantaginea flowers, Anti-inflammatory, NF-kB, Flavonoid, Phenylethanoid glycoside

*Correspondence: hjwjn2008@163.com

${ }^{2}$ Research Center of Natural Resources of Chinese Medicinal Materials and Ethnic Medicine, Jiangxi University of Chinese Medicine, No. 1688, Meiling Road, Nanchang 330004, China

Full list of author information is available at the end of the article

\section{Background}

Inflammation is an innate, automatic and complex immune system response of the body to tissue injury, infection or irritation caused by bacteria, toxins and other substances [1-4]. However, excessive inflammation 
could contribute to the pathogenesis of various acute and chronic inflammation-related diseases [1-4]. Therefore, controlling inflammatory overexpression is a vital tool for the prevention and treatment of inflammatory diseases.

Macrophages are one of the most important inflammatory and immune cells, playing a crucial role in the inflammatory process [1-4]. In particular, lipopolysaccharide (LPS) is an endotoxin that strongly triggers macrophages to activate the NF- $\mathrm{kB}$ signaling pathway and produce numerous inflammatory mediators, such as NO, TNF- $\alpha$, PGE2, IL-1 $\beta$ and IL-6 [3-7]. Therefore, the LPS-stimulated RAW 264.7 macrophage is commonly used as a classical inflammatory cell model to evaluate the anti-inflammatory activity and underlying mechanisms of action of drugs [3-7]. Furthermore, $N F-\kappa B$ is regarded as an important transcription factor in the pathogenesis of inflammatory diseases, and its activation positively regulates the expression of inflammatory mediators [3-7]. Hence, inhibition of NF-kB signaling pathway can be considered as an important target for the prevention and treatment of inflammatory diseases.

Natural products from medicinal plants, especially those derived from traditional folk medicine, are vital sources of anti-inflammatory therapy [1-7]. As one of them, Hosta plantaginea (Lam.) Aschers is an important traditional medicinal plant, mainly distributed in temperate and subtropical zones of Asia [8]. In china, the flower of $H$. plantaginea, also known as "Yu-zan-hua", has been widely used for thousands of years as a very important traditional Mongolian medicine for the treatment of inflammatory diseases, such as sore throat, acute and chronic laryngopharyngitis [8-10]. Its crude extract exhibits anti-inflammatory, antitumor, anti-viral, antimicrobial and other effects [8-10]. In our previous study, 16 flavonoids (1-16) and 3 phenylethanoid glycosides (17-19) comprising kaempferol (1), astragalin (2), kaempferol-7-O- $\beta$-D-glucopyranoside (3), kaempferol-3,7-di-O- $\beta$-D-glucopyranoside (4), kaempferol-3-O-sophoroside (5), plantanone A (6), kaempferol3 - $O-\beta$-D-[ $\beta$-D-glucopyranosyl- $(1 \rightarrow 2)$-glucopyranoside]7-O- $\beta$-D-glucopyranoside kaempferol-3-O-rutinoside-7-O-glucopyranoside kaempferol-3-O- $\alpha$-L-rhamnopyranosyl- $(1 \rightarrow 6)-\beta-\mathrm{D}$ glucopyranosyl- $(1 \rightarrow 2)-\beta$-D-glucopyranoside $(9)$ kaempferol-3-O- $\beta$-D-glucopyranosyl- $(1 \rightarrow 2)$ - $[\alpha-\mathrm{L}-$ rhamnopyranosyl- $(1 \rightarrow 6)]-\beta$-D-glucopyranoside $\quad(10)$, kaempferol-3-O-rutinoside (11), plantanone B (12), plantanone D (13), naringenin (14), dihydrokaempferol (15), hostaflavanone A (16), phenethyl-O- $\beta$-D-glucopyranoside (17), phenethanol- $\beta$-gentiobioside (18) and phenethyl$O$-rutinoside (19), were isolated from the ethanolic extract of $H$. plantaginea flowers [11-15]. Of these, all constituents except 13-15 exhibited potential inhibitory effect on cyclooxygenase $2(\mathrm{COX}-2)$ in vitro. Nevertheless, the anti-inflammatory function of these 19 constituents and their underlying mechanisms in cells have not been deeply studied.

To reveal the underlying mechanisms of $H$. plantaginea as a treatment for inflammatory diseases, the anti-inflammatory effects of these 19 constituents isolated from this traditional Chinese medicine were evaluated in LPSstimulated RAW 264.7 macrophages. We also hope to screen the most effective anti-inflammatory candidates from the flowers of $H$. plantaginea.

\section{Materials and methods}

\section{Chemicals and reagents}

Our previous studies reported the isolation and identification of 16 flavonoids and 3 phenylethanoid glycosidescomprising kaempferol (1), astragalin (2), kaempferol-7-O- $\beta$-D-glucopyranoside (3), kaempferol-3,7-di-O- $\beta$-D-glucopyranoside (4), kaempferol-3-O-sophoroside (5), plantanone A (6), kaempferol3 - $O$ - $\beta$-D-[ $\beta$-D-glucopyranosyl- $(1 \rightarrow 2)$-glucopyranoside]7-O- $\beta$-D-glucopyranoside kaempferol-3-O-rutinoside-7-O-glucopyranoside $\quad(8)$, kaempferol-3-O- $\alpha$-L-rhamnopyranosyl- $(1 \rightarrow 6)-\beta$-Dglucopyranosyl-( $1 \rightarrow 2)-\beta$-D-glucopyranoside kaempferol-3-O- $\beta$-D-glucopyranosyl- $(1 \rightarrow 2)-[\alpha-\mathrm{L}$ rhamnopyranosyl-( $(1 \rightarrow 6)]-\beta$-D-glucopyranoside $\quad(\mathbf{1 0})$, kaempferol-3-O-rutinoside (11), plantanone B (12), plantanone D (13), naringenin (14), dihydrokaempferol (15), hostaflavanone A (16), phenethyl-O- $\beta$-D-glucopyranoside (17), phenethanol- $\beta$-gentiobioside (18) and phenethyl-O-rutinoside (19) from the ethanolic extract of $H$. plantaginea flowers, a plant (Voucher specimen number: YZH201409) which was identified by professor Guoyue Zhong (Jiangxi University of Chinese Medicine, Nanchang, China) [11-15]. Moreover, the purity of each compound was greater than $97 \%$ as determined by high performance liquid chromatography analysis.

Fetal bovine serum (FBS) was obtained from Hyclone (Logan, UT, USA). Lipopolysaccharide (LPS, Escherichia coli serotype 0111: B4, L5293) was purchased from Sigma-Aldrich (St. Louis, MO, USA). Dulbecco's Modified Eagle Medium (DMEM) and trypsase were procured from GIBCO (Grand Island, NY, USA). Penicillin-streptomycin was purchased from Sigma-Aldrich (St. Louis, MO, USA). CCK-8 and radioimmunoprecipitation assay (RIPA) lysis buffer were acquired from Beyotime Institute of Biotechnology (Shanghai, China). Murine ELISA kits for TNF- $\alpha$, IL- $1 \beta$ and IL- 6 were acquired from R\&D Systems (Minnesota, USA). The murine ELISA kit for PGE2 was obtained from Westang (Shanghai, China). Antibodies against phos-NF-kB p65 
(Ser536) and NF-kB p65 were purchased from Cell Signaling Technology (Boston, USA).

\section{Cell culture}

Murine RAW 264.7 macrophages were purchased from the American Tissue Culture Collection (Manassas, USA). These cells were incubated in DMEM supplemented with $10 \% \mathrm{FBS}, 100 \mathrm{U} / \mathrm{mL}$ penicillin and $100 \mathrm{U} /$ $\mathrm{mL}$ streptomycin in humidified $5 \%$ carbon dioxide $\left(\mathrm{CO}_{2}\right)$ at $37^{\circ} \mathrm{C}[16,17]$.

\section{Effects of 19 constituents on the viability of RAW 264.7 macrophages}

The effects of 19 constituents on the viability of RAW 264.7 macrophages were assessed by CCK- 8 assay [18, 19]. Prior to treatment, RAW 264.7 macrophages $\left(5 \times 10^{3}\right.$ cells/well) were seeded into 96 -well plates and incubated for $24 \mathrm{~h}$. All cultured cells were treated with or without 19 compounds at a concentration of $40 \mu \mathrm{M}$ at $37^{\circ} \mathrm{C}$ for $24 \mathrm{~h}$. After incubation, $10 \mu \mathrm{L}$ of CCK- 8 solution was added to each well and incubated at $37^{\circ} \mathrm{C}$. After $2 \mathrm{~h}$, the absorbance of each well was measured at $450 \mathrm{~nm}$ in a microplate reader. Cell viability was calculated using the following formular:

Cell viability $(\%)=\mathrm{A}_{\text {sample }} / \mathrm{A}_{\text {control }} \times 100$, where $\mathrm{A}_{\text {sample }}$ and $A_{\text {control }}$ are the absorbance of cells treated with the compound and untreated cells, respectively. Moreover, the latter is expressed as $100 \%$ cell viability.

\section{Effects of 19 constituents on LPS-induced NO production by the Griess method}

The effects of 19 constituents on NO production in LPS-induced RAW 264.7 macrophages were determined using the Griess method [18, 19]. Prior to treatment, RAW 264.7 macrophages $\left(5 \times 10^{3}\right.$ cells/ well) were seeded into 96-well plates and incubated for $24 \mathrm{~h}$. All cultured cells were treated with or without 19 compounds at a concentration of $40 \mu \mathrm{M}$ at $37^{\circ} \mathrm{C}$. After $1 \mathrm{~h}$, cells were incubated with or without LPS at a concentration of $1 \mu \mathrm{g} / \mathrm{mL}$ at $37^{\circ} \mathrm{C}$ for $24 \mathrm{~h}$. After incubation, $50 \mu \mathrm{L}$ of each supernatant solution was collected and mixed with equal volumes of Griess Reagent I and Griess Reagent II, respectively. The absorbance (A) was measured at $540 \mathrm{~nm}$ after $10 \mathrm{~min}$ of incubation at room temperature. The standard concentration of sodium nitrite was used to calculate the nitrite concentration. Moreover, the $\mathrm{NO}$ inhibition rate

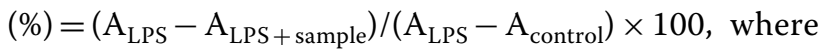
$\mathrm{A}_{\mathrm{LPS}}, \mathrm{A}_{\mathrm{LPS}+\text { sample, }}$ and $\mathrm{A}_{\text {control }}$ are the absorbance of LPS model group, LPS + sample group, and DMEM group, respectively.

Subsequently, all compounds with NO inhibition rates greater than 50\%, including 1, 4-7, 11, 13-15 and 17, were further investigated in accordance with the method described above to determine the NO levels at concentrations of $1.25,2.5,5,10$ and $20 \mu \mathrm{M}$, respectively.

\section{Effects of compounds 1, 4-6, 15 and 17 on LPS-induced pro-inflammatory cytokine production by ELISA assay} The logarithmic growth phase of RAW 264.7 macrophages was inoculated in 96-well plates at a density of $5 \times 10^{3}$ cells/well. After $24 \mathrm{~h}$ of incubation, these cells were pretreated with respective concentrations of $20 \mu \mathrm{M}$ of each compound (1, 4-6, 15 and 17) for $1 \mathrm{~h}$, followed by the addition of LPS $(1 \mu \mathrm{g} / \mathrm{mL})$ for $24 \mathrm{~h}$. Finally, $50 \mu \mathrm{L}$ of each supernatant solution was taken to measure the concentrations of TNF- $\alpha$, PGE2, IL- $1 \beta$ and IL- 6 , using the corresponding commercially available murine ELISA kits in accordance with the manufacturer's instructions $[16,17]$.

\section{Effects of compounds 1, 4-6, 15, and 17 on LPS-induced NF-kB activation by Western blotting}

RAW 264.7 macrophages were seeded and pretreated with respective concentrations of $20 \mu \mathrm{M}$ of each compound $(1,4-6,15$ and 17) for $1 \mathrm{~h}$, followed by the addition of LPS $(1 \mu \mathrm{g} / \mathrm{mL})$ for $24 \mathrm{~h}$. Subsequently, the total protein was extracted by resuspending the cells in RIPA lysis buffer. In addition, protein concentrations were measured by a Bicinchoninic acid (BCA) assay kit. Proteins were separated using SDS-PAGE gels and then electroplated onto a PVDF membrane, which was blocked with $5 \%$ skim milk for $1 \mathrm{~h}$ at room temperature in Trisbuffered saline-Tween (TBST). Membranes were washed three times with TBST and then incubated overnight at $4{ }^{\circ} \mathrm{C}$ in diluted $(1: 1000)$ primary antibody solution (antiNF-kB p65 or anti-phosphorylated-NF-kB p65). After washing three times with TBST, the membranes were incubated with a 1:5000 dilution of HRP-conjugated secondary antibody for $1 \mathrm{~h}$ at room temperature. The immunoreactive bands were determined by densitometry and quantified using a Bio-Rad auto-developer (Bio-Rad, California, USA). All results are expressed as relative ratios to the reference protein GAPDH $[16,17]$.

\section{Statistical analysis}

All results were reproduced in triplicate and expressed as mean \pm standard deviation (SD). Multiple data sets were compared using one-way analysis of variance (ANOVA) followed by Tukey's test using GraphPad Prism 6, and $P<0.05$ was considered significant.

\section{Results}

Absence of negative effect of all constituents on RAW 264.7 macrophage viability

The cell viability of these 19 constituents in RAW 264.7 macrophages was performed using the CCK-8 method. 
As shown in Table 1, all constituents at a concentration of $40 \mu \mathrm{M}$ showed no toxicity to RAW 264.7 macrophages after $24 \mathrm{~h}$ of treatment $(p>0.05)$. Accordingly, subsequent experiments were conducted with 19 constituents at concentrations not exceeding $40 \mu \mathrm{M}$.

\section{Reduction of NO production in LPS-a-induced RAW 264.7 macrophages by all constituents}

As illustrated in Fig. 1, LPS induced a dramatic production of $\mathrm{NO}$, which was prominently reduced by 19 constituents at a concentration of $40 \mu \mathrm{M}$, and their NO

Table 1 Effects of 16 flavonoids (1-16) and 3 phenylethanoid glycosides (17-19) on cell viability of RAW 264.7 macrophages $^{\text {a }}$

\begin{tabular}{llll}
\hline Compound & Cell viability (\%) & Compound & Cell viability (\%) \\
\hline Control & $100 \pm 10.32$ & $\mathbf{1 0}$ & $93.76 \pm 5.06$ \\
$\mathbf{1}$ & $92.36 \pm 3.96$ & $\mathbf{1 1}$ & $90.87 \pm 5.45$ \\
$\mathbf{2}$ & $95.01 \pm 1.86$ & $\mathbf{1 2}$ & $91.93 \pm 8.04$ \\
$\mathbf{3}$ & $91.12 \pm 5.28$ & $\mathbf{1 3}$ & $91.84 \pm 1.11$ \\
$\mathbf{4}$ & $93.38 \pm 6.34$ & $\mathbf{1 4}$ & $92.97 \pm 6.19$ \\
$\mathbf{5}$ & $93.48 \pm 5.33$ & $\mathbf{1 5}$ & $91.31 \pm 5.38$ \\
$\mathbf{6}$ & $96.89 \pm 1.04$ & $\mathbf{1 6}$ & $92.56 \pm 1.97$ \\
$\mathbf{7}$ & $94.03 \pm 4.52$ & $\mathbf{1 7}$ & $92.21 \pm 3.48$ \\
$\mathbf{8}$ & $95.48 \pm 3.54$ & $\mathbf{1 8}$ & $92.51 \pm 5.62$ \\
$\mathbf{9}$ & $90.70 \pm 3.71$ & $\mathbf{1 9}$ & $92.30 \pm 9.38$ \\
\hline
\end{tabular}

a Values are mean \pm SD of three independent experiments $(n=3)$. One-way ANOVA followed by Tukey's test with GraphPad Prism 6 inhibition rates exceeded $50 \%$ except for compounds 2 , $3,8-10,12,16,18$ and 19.

Subsequently, 9 flavonoids $(1,4-7,11$ and 13-15) and one phenylethanoid glycoside (17) were further evaluated for their inhibitory effects on NO production in LPSstimulated RAW 264.7 macrophages. As a result, these ten constituents may remarkably reduce NO production in a concentration-dependent manner with half-maximal inhibitory concentration $\left(\mathrm{IC}_{50}\right)$ values in the range of $12.20-38.53 \mu \mathrm{M}$ (Table 2 and Fig. 2). Among them, compounds 1, 4-6, 15 and 17 showed the strongest effect on NO production with $\mathrm{IC}_{50}$ values not exceeding $20 \mu \mathrm{M}$. As such, compounds 1, 4-6, 15 and 17 were further evaluated for their anti-inflammatory effects and underlying mechanisms in LPS-stimulated RAW 264.7 macrophages.

Based on the above results, 9 flavonoids comprising 1, 47,11 and 13-15, as well as one phenylethanoid (17), were identified as the bioactive phytochemicals contributing to anti-inflammatory activity against NO production in LPSstimulated RAW 264.7 macrophages. Importantly, compounds 1, 4-6, 15 and 17 showed the highest efficacy against $\mathrm{NO}$ inhibition with $\mathrm{IC}_{50}$ values less than $20 \mu \mathrm{M}$, and were further chosen to explore the anti-inflammatory mechanism.

\section{Inhibiting the release of TNF- $\alpha$, PGE2, IL-1 $\beta$ and IL- 6 in LPS-stimulated RAW 264.7 macrophages by compounds} $1,4-6,15$ and 17

To determine whether compounds 1, 4-6, 15 and 17 affected the secretion of pro-inflammatory cytokines

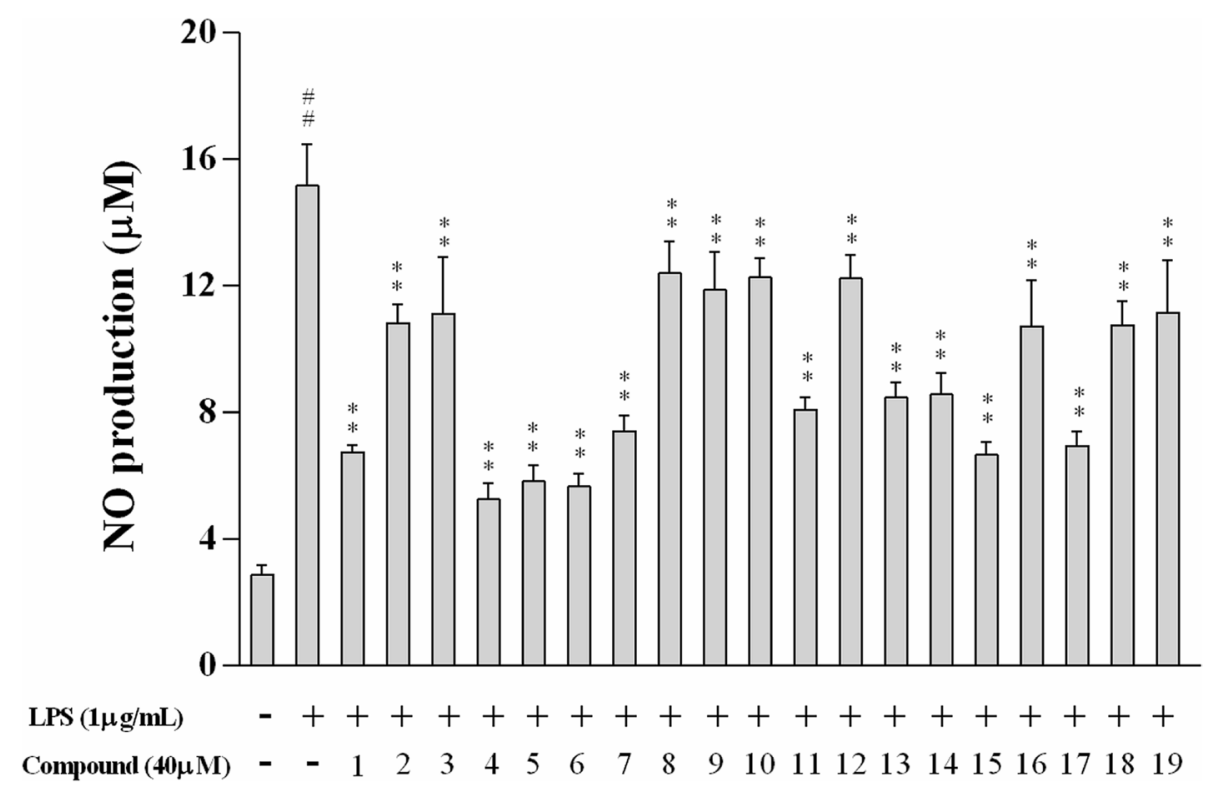

Fig. 1 Effects of 16 flavonoids (1-16) and 3 phenylethanoid glycosides (17-19) on NO production in LPS-stimulated RAW 264.7 macrophages. All data from three independent experiments are expressed as mean \pm SD. ${ }^{\# \#} p<0.01$ vs. culture medium-only control group; ${ }^{* *} p<0.01$ vs. LPS-only model group. One-way ANOVA, followed by Tukey's test using GraphPad Prism 6 
Table 2 The $I C_{50}$ values of 16 flavonoids (1-16) and 3 phenylethanoid glycosides (17-19) on NO production in LPSstimulated RAW 264.7 macrophages $^{a}$

\begin{tabular}{llll}
\hline Compound & $\mathrm{IC}_{\mathbf{5 0}}(\boldsymbol{\mu M})$ & Compound & $\mathrm{IC}_{\mathbf{5 0}}(\boldsymbol{\mu M})$ \\
\hline $\mathbf{1}$ & $18.42 \pm 2.67$ & $\mathbf{1 1}$ & $31.18 \pm 1.09$ \\
$\mathbf{2}$ & $>40$ & $\mathbf{1 2}$ & $>40$ \\
$\mathbf{3}$ & $>40$ & $\mathbf{1 3}$ & $36.15 \pm 3.77$ \\
$\mathbf{4}$ & $12.20 \pm 1.18$ & $\mathbf{1 4}$ & $38.53 \pm 2.78$ \\
$\mathbf{5}$ & $13.09 \pm 1.61$ & $\mathbf{1 5}$ & $18.34 \pm 1.71$ \\
$\mathbf{6}$ & $12.62 \pm 0.76$ & $\mathbf{1 6}$ & $>40$ \\
$\mathbf{7}$ & $26.52 \pm 1.53$ & $\mathbf{1 7}$ & $19.91 \pm 2.01$ \\
$\mathbf{8}$ & $>40$ & $\mathbf{1 8}$ & $>40$ \\
$\mathbf{9}$ & $>40$ & $\mathbf{1 9}$ & $>40$ \\
$\mathbf{1 0}$ & $>40$ & & \\
\hline
\end{tabular}

${ }^{a}$ Values are mean \pm SD of three independent experiments $(n=3)$ (including TNF- $\alpha$, PGE2, IL-1 $\beta$ and IL-6) in LPS-stimulated RAW 264.7 macrophages, an ELISA method was performed. As depicted in Fig. 3, the levels of TNF- $\alpha$, PGE2, IL-1 $\beta$ and IL- 6 were prominently increased after LPS $(1 \mu \mathrm{g} / \mathrm{mL})$ treatment compared to the control group $(p<0.01)$. In contrast, treatment with compounds $\mathbf{1}$, 4-6, 15 and 17 at a concentration of $20 \mu \mathrm{M}$ significantly reduced the levels of TNF- $\alpha$, PGE2, IL-1 $\beta$ and IL- 6 compared to the LPS group $(p<0.01)$.

\section{Blocking NF-kB signaling pathway in LPS-stimulated RAW} 264.7 macrophages by compounds 1, 4-6, 15 and 17

To determine the underlying anti-inflammatory mechanism of compounds $1,4-6,15$ and 17 , the protein levels of NF-kB p65 and phosphorylated NF-kB p65 were examined by Western blot analysis. As shown in Fig. 4, the protein expression of phosphorylated
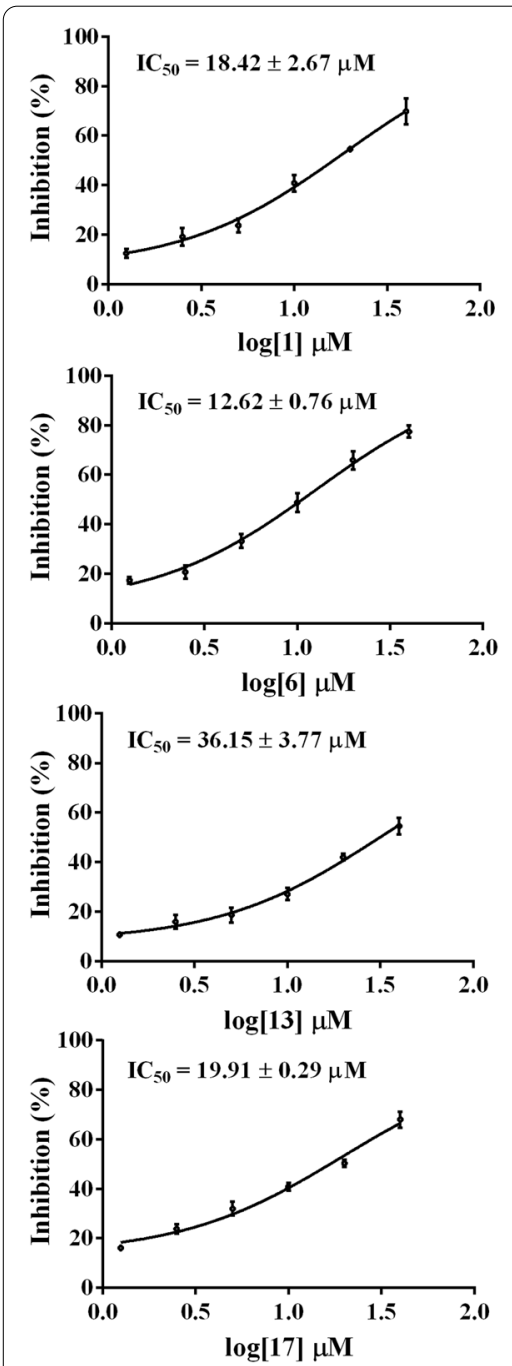
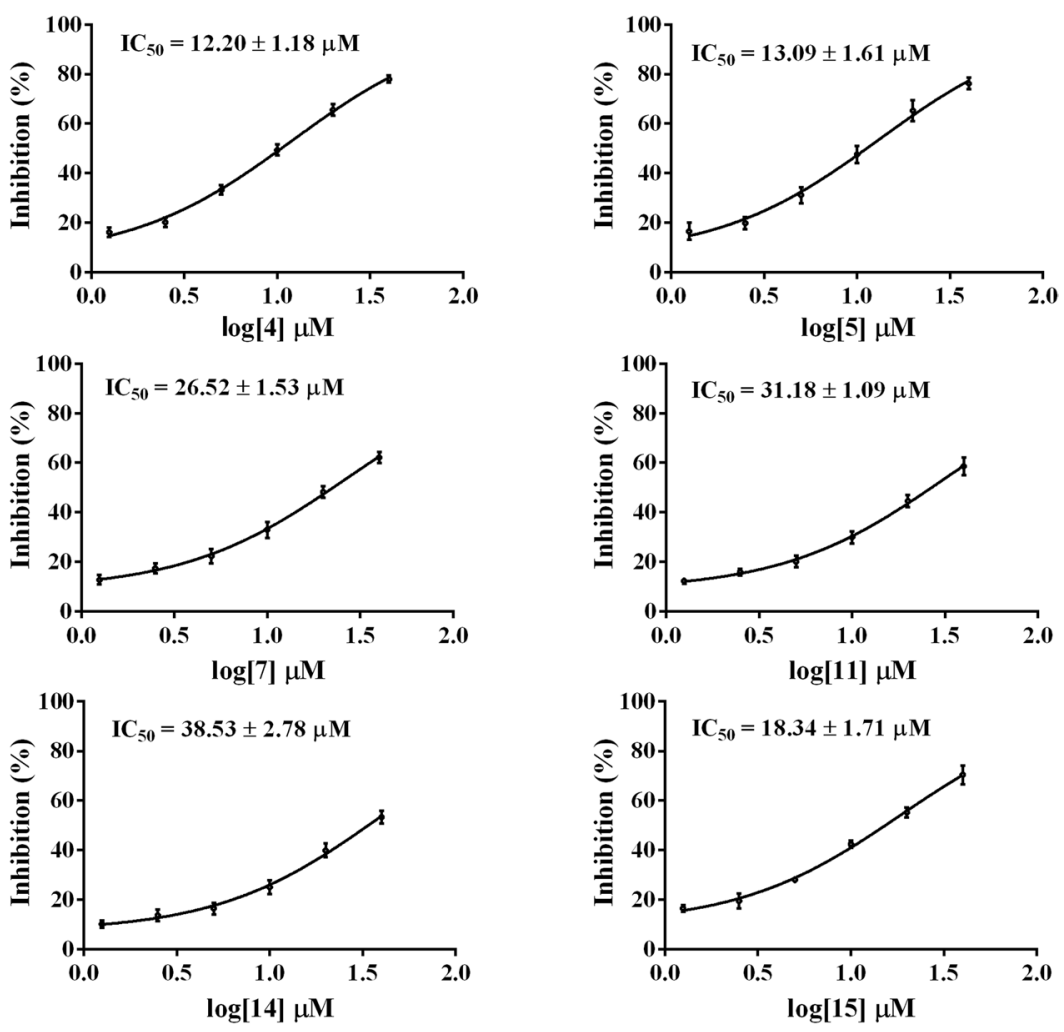

Fig. 2 Dose-response curves for compounds 1, 4-7, 11, 13-15 and $\mathbf{1 7}$ in the NO inhibition assay $(n=3)$ 

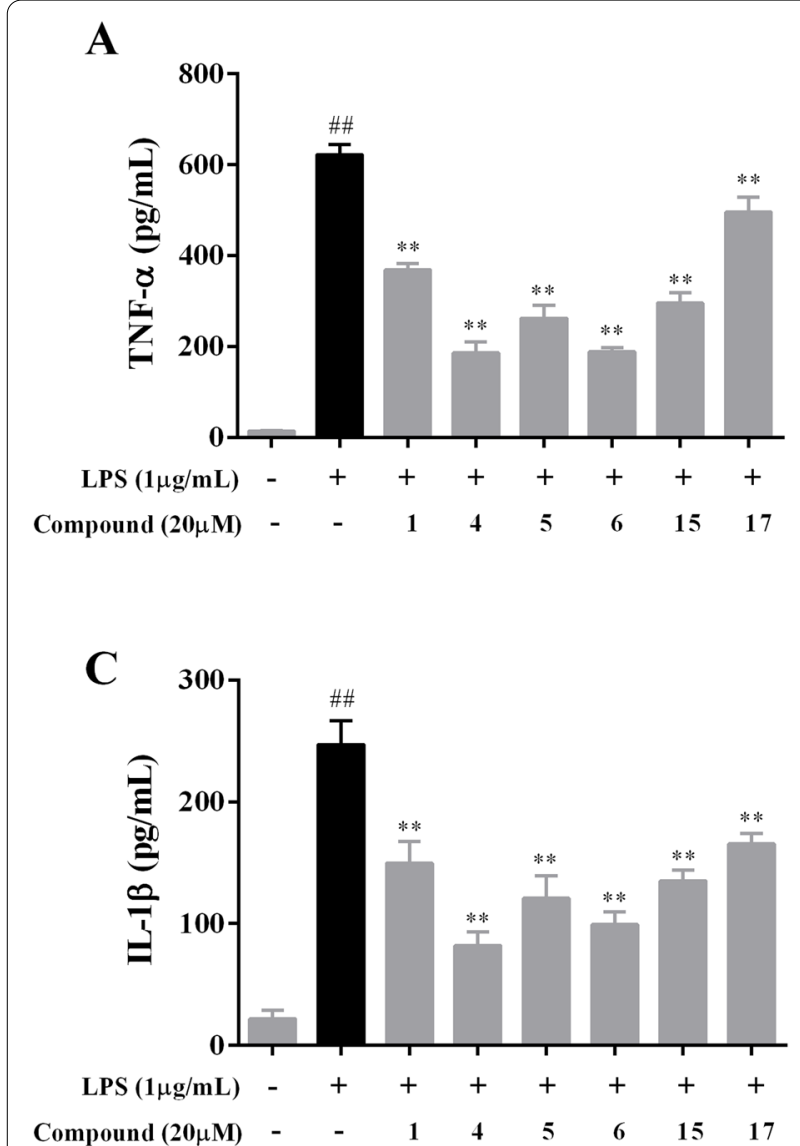
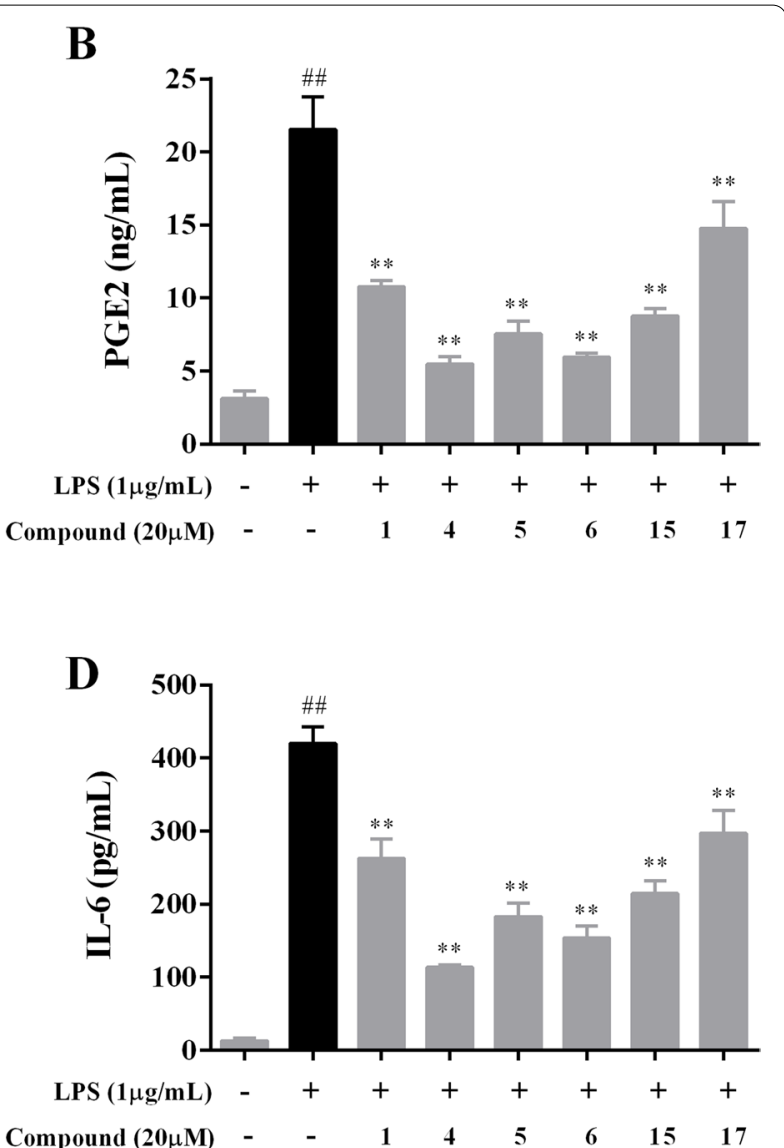

Fig. 3 Effects of compounds 1, 4-6, 15 and $\mathbf{1 7}$ on the production of TNF-a (A), PGE2 (B), IL-1 $\beta$ (C) and IL-6 (D) in LPS-stimulated RAW 264.7 macrophages. All data from three independent experiments are expressed as mean $\pm \mathrm{SD}$. ${ }^{\#} p<0.01$ vs. culture medium-only control group; ${ }^{* *} p<0.01$ vs. LPS-only model group. One-way ANOVA, followed by Tukey's test using GraphPad Prism 6

NF-kB p65 was significantly upregulated in LPS-treated RAW 264.7 macrophages compared to untreated cells $(p<0.01)$. Phosphorylation of NF-kB p65 levels was found to be significantly lower in the treatment groups of compounds 1, 4-6, 15 and 17 at a concentration of $20 \mu \mathrm{M}$ compared to the LPS group $(p<0.01)$ (Supplementary Material).

Based on the above evidence, compounds 1, 4-6, 15 and $\mathbf{1 7}$ are the most potent anti-inflammatory constituents that suppress the NF- $\mathrm{kB}$ signaling pathway, which results in a reduction in the secretion levels of NO, TNF$\alpha$, PGE2, IL-1 $\beta$ and IL-6 in LPS-stimulated RAW 264.7 macrophages.

\section{Discussion}

In China, the flower of $H$. plantaginea is commonly used as an empirical treatment for inflammatory diseases with very limited scientific validation [8]. Crude extracts of $H$. plantaginea have been evaluated for their traditional pharmacological effects such as anti-inflammatory, anti-tumor, anti-viral, antimicrobial effects, etc. [8-10].
Although numerous phytochemicals with anti-inflammatory, anti-tumor, anti-acetylcholinesterase, and anti-viral activities have been reported [8,9], the anti-inflammatory effects and underlying mechanisms of action of constituents derived from the flowers of $H$. plantaginea have not been fully explored. Furthermore, flavonoids and phenylethanoid glycosides are two major classes of phytochemicals from medicinal plants with various biological effects, such as anti-inflammatory and antioxidant $[1$, $11,13,20]$. In our previous studies, 16 flavonoids (1-16) and 3 phenylethanoid glycosides (17-19), some of which have potential anti-inflammatory activity against COX-2, were isolated and identified from the ethanolic extract of H. plantaginea flowers [11-15]. Furthermore, COX-2 is a critical enzyme involved in the process of inflammatory responses and inflammatory diseases [11,21-28], which suggests that the aforementioned 19 constituents may have anti-inflammatory effects. Although kaempferol (compound 1) [29, 30], astragalin (compound 2) [30], kaempferol-3-O-rutinoside (compound 11) [31], and dihydrokaempferol (compound 15) [32] have been shown 


\section{A}
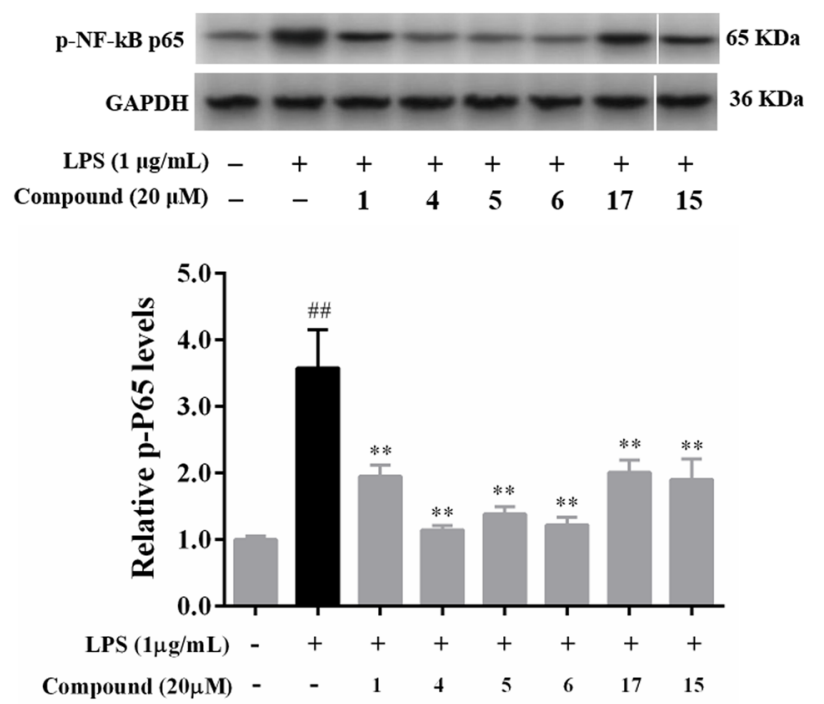

B
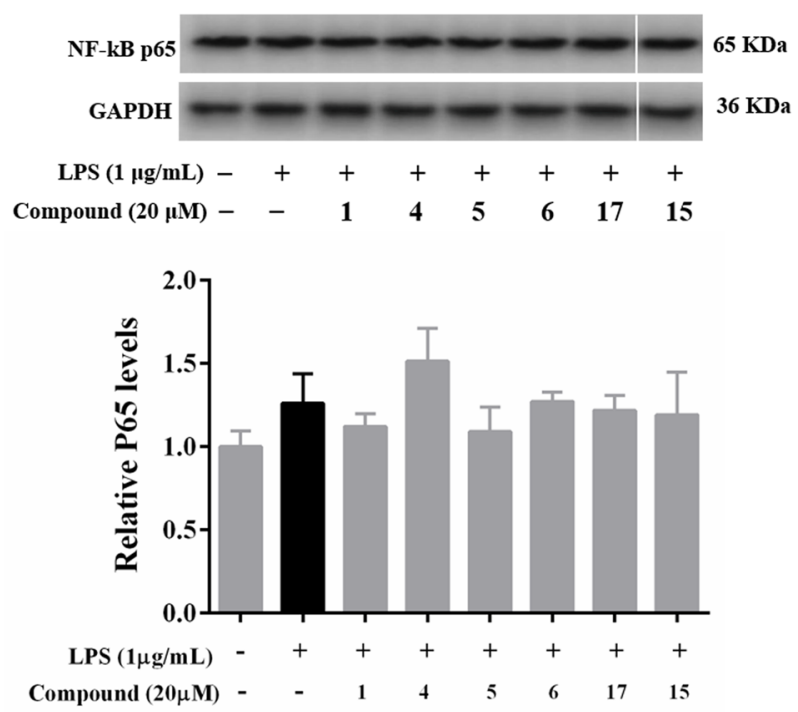

Fig. 4 Effects of compounds 1, 4-6, 15 and $\mathbf{1 7}$ on NF-kB activation (A-phosphorylated p65 and B-total p65) in LPS-stimulated RAW 264.7 macrophages. All data from three independent experiments are expressed as mean \pm SD. ${ }^{\#} p<0.01$ vs. culture medium-only control group; ${ }^{* *} p<0.01$ vs. LPS-only model group. One-way ANOVA, followed by Tukey's test using GraphPad Prism 6

to exhibit anti-inflammatory effects by suppressing the secretion of inflammatory cytokines in cells, their underlying molecular mechanisms remain unclear. Furthermore, naringenin (compound 14) at concentrations of 40, 60 and $80 \mu \mathrm{M}$ possessed anti-inflammatory effect via inhibition NF- $\mathrm{kB}$ and MAPKs pathways in BV2 microglia [33]. Taken together, the anti-inflammatory effects of these 19 constituents isolated from $H$. plantaginea flowers and their underlying mechanisms are still poorly understood in cellular model.

In the present study, the anti-inflammatory activities of 19 constituents isolated from $H$. plantaginea flowers were evaluated in LPS-stimulated RAW 264.7 macrophages. As a result, 5 flavonoids (1, 4-6 and 15) and one phenylethanoid glycoside (17) exhibited strong antiinflammatory effects by blocking the NF-kB signaling pathway and suppressing NO, TNF- $\alpha$, PGE2, IL- $1 \beta$ and IL-6 production at a concentration of $20 \mu \mathrm{M}$.

LPS promotes the inflammatory process, and LPSstimulated RAW 264.7 macrophages have been widely used in inflammation studies [1-4]. Several inflammatory mediators such as NO, TNF- $\alpha$, PGE2, IL- $1 \beta$ and IL-6, as well as the NF- $\mathrm{kB}$ signaling pathway, are closely associated with inflammatory diseases $[2,4,6,28]$. In addition, stimulation of LPS can lead to activation of the NF-kB signaling pathway and result in the production of numerous inflammatory mediators [3-6, 25, 26]. Therefore, suppressing the inflammatory response and reducing the production of inflammatory mediators may be a pivotal strategy for the prevention and treatment of various inflammatory diseases.

NO, produced from eNOS and iNOS, is an important and classic biomarker of inflammation [16, 17]. As one of the most crucial inflammatory mediators, excessive production of $\mathrm{NO}$ is an important feature of the inflammatory response of LPS-stimulated RAW 264.7 macrophages [14]. In particular, excessive secretion of $\mathrm{NO}$ stimulates the activation of NF- $\mathrm{kB}$ and other signaling pathways, which leads to the over-secretion of NO, TNF- $\alpha$, PGE2, IL-1 $\beta$, IL-6 and other pro-inflammatory cytokines [3-6, 25, 26]. Therefore, inhibiting the overproduction of $\mathrm{NO}$ is a vital tool for anti-inflammatory agents. The results of this study showed 19 constituents isolated from $H$. plantaginea flowers significantly suppressed the overproduction of NO in LPS-stimulated RAW 264.7 macrophages. Among them, 5 flavonoids (1, 4-6 and 15) and one phenylethanoid glycoside (17) exhibited the most significant effect on NO production with $\mathrm{IC}_{50}$ values in the range of $12.20-19.91 \mu \mathrm{M}$. Comparing the structures of flavonoids and their antiinflammatory effects, all anti-inflammatory flavonoids contained either zero or two glycosyls except compound 7 , and kaempferol derivatives containing only glucosyls showed stronger anti-inflammatory activities than others belonging to flavonol glycosides, suggesting that glycosylation of certain cites and numbers may contribute to the anti-inflammatory activities of kaempferol. Although many compounds counteract the production of $\mathrm{NO}$ in cells, it remains unclear whether eNOS and/or iNOS induce NO. 
There is substantial evidence that the massive production of pro-inflammatory cytokines, such as TNF- $\alpha$, PGE2, IL-1 $\beta$ and IL-6, is closely associated with inflammatory diseases $[3-6,23,24]$. TNF- $\alpha$ participates in the regulation of inflammation and is involved in several inflammatory diseases. Moreover, PGE2 is an important pro-inflammatory mediator that plays a critical role in the course of the inflammatory response. Similarly, IL-1 $\beta$ and IL-6 play a very important role in inflammation. Therefore, suppression of TNF- $\alpha$, PGE2, IL- $1 \beta$ and IL- 6 production seems to be a very effective method to inhibit the abnormal inflammatory response. In this work, compounds 1, 4-6, 15 and 17 remarkably suppressed the production of TNF- $\alpha$, PGE2, IL- $1 \beta$ and IL- 6 in LPSactivated RAW 264.7 macrophages at a concentration of $20 \mu \mathrm{M}$.

Numerous studies have reported that NF-kB is a key transcription factor in the pathogenesis of inflammatory diseases and its activation positively regulates the expression of inflammatory mediators such as NO, TNF- $\alpha$, PGE2, IL-1 $\beta$ and IL-6 [3-6, 23, 24]. Furthermore, NF- $\mathrm{kB}$ consists primarily of p50 and p 65 subunits, the latter of which responds to pro-inflammatory cytokine stimulation [5, 28]. Thus, suppressing NF-kB p65 translocation to the nucleus is considered a key target and an effective therapeutic strategy for the treatment of inflammatory diseases. In this study, compounds 1, 4-6, 15, and 17 prominently prevented the phosphorylation of $\mathrm{p} 65$ translocation, resulting in the blockade of NF- $\mathrm{kB}$ subunit $\mathrm{p} 65$ nuclear translocation in RAW 264.7 macrophages. These results suggest that the inhibitory effect of these constituents on the NF- $\mathrm{kB}$ signaling pathway reduces the levels of inflammatory cytokines, including NO, TNF- $\alpha$, PGE2, IL-1 $\beta$ and IL-6.

\section{Conclusions}

In conclusion, 5 flavonoids $(\mathbf{1}, 4-6$ and 15$)$ and one phenylethanoid glycoside (17), especially 4-6 derived from the flowers of $H$. plantaginea, exerted significant antiinflammatory effects by inhibiting the NF- $\mathrm{kB}$ signaling pathway and suppressing NO, TNF- $\alpha$, PGE2, IL- $1 \beta$ and IL-6 in LPS-stimulated RAW 264.7 macrophages. The present study strongly supports the use of $H$. plantaginea flowers as a novel candidate for anti-inflammatory therapy. In addition, these flavonoids and phenylethanoid glycoside may be candidates for the management of inflammatory diseases.

\footnotetext{
Abbreviations

ANOVA: One-way analysis of variance; CCK-8: Cell Counting Kit-8; $\mathrm{CO}_{2}$ : Carbon dioxide; DMEM: Dulbecco's Modified Eagle's Medium; ELISA: Enzyme-linked immunosorbent assay; FBS: Fetal bovine serum; LPS: Lipopolysaccharide; $I_{50}$ : Half maximal inhibitory concentration; IL-1 $\beta$ : Interleukin 1 $\beta$; NO: Nitric oxide; NF-kB: Nuclear factor kappa B; PGE2: Prostaglandin E2; RIPA: Radioimmunoprecipitation assay; SD: Standard deviation; TNF-a: Tumor necrosis factor a.
}

\section{Supplementary Information}

The online version contains supplementary material available at https://doi. org/10.1186/s12906-022-03540-1.

Additional file 1.

Acknowledgments

Not applicable.

\section{Authors' contributions}

$\mathrm{JH}$ designed the study. $\mathrm{JH}$ and LY carried out most experiments, analyzed the data and wrote the manuscript. All authors read and approved the final manuscript.

\section{Funding}

This work was supported by Jiangxi University of Chinese Medicine (Nos. JXXT2017001 and s202010412068).

Availability of data and materials

The data used to support the findings of this study are available from the corresponding author upon request.

\section{Declarations}

Ethics approval and consent to participate

Not applicable.

\section{Consent for publication}

Not applicable.

\section{Competing interests}

The authors declare no competing interests.

\section{Author details}

${ }^{1}$ College of Pharmacy, Jiangxi University of Chinese Medicine, Nanchang 330004, China. ${ }^{2}$ Research Center of Natural Resources of Chinese Medicinal Materials and Ethnic Medicine, Jiangxi University of Chinese Medicine, No. 1688, Meiling Road, Nanchang 330004, China.

Received: 7 July 2021 Accepted: 23 February 2022

Published online: 03 March 2022

\section{References}

1. Zhao XL, Yu L, Zhang SD, Ping K, Ni HY, Qin XY, et al. Cryptochlorogenic acid attenuates LPS-induced inflammatory response and oxidative stress via upregulation of the $\mathrm{Nrf2} / \mathrm{HO}-1$ signaling pathway in RAW 264.7 macrophages. Int Immunopharmacol. 2020;83:106436.

2. Liu SM, Yang TC, Ming TW, Gaun TKW, Zhou T, Wang S, et al. Isosteroid alkaloids with different chemical structures from Fritillariae cirrhosae bulbus alleviate LPS-induced inflammatory response in RAW 264.7 cells by MAPK signaling pathway. Int Immunopharmacol. 2020;78:106047.

3. Karunarathne WAHM, Lee KT, Choi YH, Jin CY, Kim GY. Anthocyanins isolated from Hibiscus syriacus L. attenuate lipopolysaccharide-induced inflammation and endotoxic shock by inhibiting the TLR4/MD2-mediated NF-KB signaling pathway. Phytomedicine. 2020;76:153237.

4. Lee HH, Jang E, Kang SY, Shin JS, Han HS, Kim TW, et al. Anti-inflammatory potential of Patrineolignan B isolated from Patrinia scabra in LPS-stimulated macrophages via inhibition of NF-KB, AP-1, and JAK/STAT pathways. Int Immunopharmacol. 2020:86:106726.

5. Wang LY, Gu JP, Zong MY, Zhang QR, Li HX, Li DN, et al. Anti-inflammatory action of physalin $\mathrm{A}$ by blocking the activation of NF-KB signaling pathway. J Ethnopharmacol. 2021;267:113490.

6. Linghu KG, Ma QS, Zhao GD, Xiong W, Lin LG, Zhang QW, et al. Leocarpinolide B attenuates LPS-induced inflammation on RAW264.7 macrophages by mediating NF-KB and Nrf2 pathways. Eur J Pharmacol. 2020;868:172854. 
7. Jeong DE, Shim SY, Lee M. Anti-inflammatory activity of phenylpropyl triterpenoids from Osmanthus fragrans var. aurantiacus leaves. Int Immunopharmacol. 2020;86:106576.

8. Yang L, He JW. Traditional uses, phytochemistry, pharmacology and toxicological aspects of the genus Hosta (Liliaceae): a comprehensive review. J Ethnopharmacol. 2021;265:113323.

9. Yang L, He JW. Hosta plantaginea (Lam.) Aschers (Yuzan): an overview on its botany, traditional use, phytochemistry, quality control and pharmacology. RSC Adv. 2019:9:35050-8.

10. He JW, Wu LF, Yang L, Zhao BY, Li CL. Extraction of phenolics and flavonoids from four Hosta species using reflux and ultrasound-assisted methods with antioxidant and a-glucosidase inhibitory activities. Biomed Res Int. 2020:2020:6124153.

11. He JW, Yang L, Mu ZQ, Zhu YY, Zhong GY, Liu ZY, et al. Anti-inflammatory and antioxidant activities of flavonoids from the flowers of Hosta plantaginea. RSC Adv. 2018;8:18175-9.

12. Yang L, Lin YM, He ZW, Zhang TF, Li Y, Xie XT, et al. Hostaflavanol A, a new anti-inflammatory and antioxidant activities flavanol from the flowers of Hosta plantaginea. Med Chem Res. 2020;29:426-30.

13. Yang $L$, Jiang ST, Zhou QG, Zhong GY, He JW. Chemical constituents from the flower of Hosta plantaginea with cyclooxygenases inhibition and antioxidant activities and their chemotaxonomic significance. Molecules. 2017;22:1825.

14. He JW, Huang XY, Wang YQ, Liang J, Liu RH, Zhong GY, et al. A new flavonol glycoside from the flowers of Hosta plantaginea with cyclooxygenases-1/2 inhibitory and antioxidant activities. Nat Prod Res. 2019:33:1599-604.

15. Yang $L$, Zhu $Y Y$, He ZW, Zhang TF, Xiao ZX, Xu RL, et al. Plantanone $D$, a new rare methyl-flavonoid from the flowers of Hosta plantaginea with anti-inflammatory and antioxidant activities. Nat Prod Res. 2021:35:4331-7.

16. Fang YW, Yang L, He JW. Plantanone $C$ attenuates LPS-stimulated inflammation by inhibiting NF-kB/iNOS/COX-2/MAPKs/Akt pathways in RAW 264.7 macrophages. Biomed Pharmacother. 2021;143:112104.

17. Yang L, Cao L, Li CL, Li XB, Wang JS, Chen HP, et al. Hostaflavone A from Hosta plantaginea (lam.) Asch. Blocked NF-kB/iNOS/COX-2/MAPKs/Akt signaling pathways in LPS-induced RAW 264.7 macrophages. J Ethnopharmacol. 2022;282:114605.

18. Yang L, Liu RH, Fang YW, He JW. Anti-inflammatory effect of phenylpropanoids from Dendropanax dentiger in TNF-a-induced MH7A cells via inhibition of NF-KB, Akt and JNK signaling pathways. Int Immunopharmacol. 2021;94:107463.

19. Yang L, Liu RH, Ouyang S, Zou M, Duan YL, Li LM, et al. Compounds DRG and DAG, two phenol glycosides inhibit TNF-a-stimulated inflammatory response through blocking NF-KB/AKT/JNK signaling pathways in MH7A cells. Inflammation. 2021;44:1762-70.

20. Wu AZ, Yang ZY, Huang YM, Yuan H, Lin CZ, Wang T, et al. Natural phenylethanoid glycosides isolated from Callicarpa kwangungensis suppressed lipopolysaccharide-mediated inflammatory response via activating Keap1/Nrf2/HO-1 pathway in RAW 264.7 macrophages cell. J Ethnopharmacol. 2020;258:112857.

21. Yang L, Liu SZ, Liu RH, He JW. Bioassay-guided isolation of cyclooxygenase-2 inhibitory and antioxidant phenylpropanoid derivatives from the roots of Dendropanax dentiger. Bioorg Chem. 2020;104:104211.

22. Yang L, Liu RH, Fan AG, Zhong GY, He JW. Dendropanax dentiger (Harms) Merr. root and its major constituents exert therapeutic effect on adjuvant-induced arthritis in rats. J Ethnopharmacol. 2021;267:113631.

23. Yang L, Liu RH, Fan AG, Zhao JJ, Zhang Y, He JW. Chemical composition of Pterospermum heterophyllum root and its anti-arthritis effect on adjuvantinduced arthritis in rats via modulation of inflammatory responses. Front Pharmacol. 2020;11:584849.

24. Yang L, Fang YW, Liu RH, He JW. Phytochemical analysis, anti-inflammatory and antioxidant activities of Dendropanax dentiger roots. Biomed Res Int. 2020;2020:5084057.

25. Xu XX, Gao HW, Hou Y, Yu J, Sun W, Wang Y, et al. Dihydronortanshinone, a natural product, alleviates LPS-induced inflammatory response through NF-KB, mitochondrial ROS, and MAPK pathways. Toxicol Appl Pharmacol. 2018:355:1-8

26. Ren J, Li LX, Wang Y, Zhai JC, Chen GT, Hu K. Gambogic acid induces heme oxygenase-1 through Nrf2 signaling pathway and inhibits NF-KB and MAPK activation to reduce inflammation in LPS-activated RAW 264.7 cell. Biomed Pharmacother. 2019;109:555-62.

27. Park SB, Park GH, Kim HN, Song HJ, Song HM, Kim HS, et al. Anti-inflammatory effect of the extracts from the branch of Taxillus yadoriki being parasitic in Neolitsea sericea in LPS-stimulated RAW 264.7 cells. Biomed Pharmacother. 2018;104:1-7.

28. Han JM, Lee EK, Gong SY, Sohng JK, Kang YJ. Sparassis crispa exerts anti-inflammatory activity via suppression of TLR-mediated NF-KB and MAPK signaling pathways in LPS-induced RAW 264.7 macrophage cells. J Ethnopharmacol. 2019;231:10-8.

29. Harasstani OA, Moin S, Tham CL, Liew CY, Ismail N, Rajajendram R, et al. Flavonoid combinations cause synergistic inhibition of inflammatory mediator secretion from lipopolysaccharide-induced RAW 264.7 cells. Inflamm Res. 2010;59:711-21.

30. Wang J, Mazza G. Effects of anthocyanins and other phenolic compounds on the production of tumor necrosis factor a in LPS/IFN- $\gamma$-activated RAW 264.7 macrophages. J Agric Food Chem. 2002;50:4183-9.

31. Wang GJ, Chen YM, Wang TM, Lee CK, Chen KJ, Lee TH. Flavonoids with iNOS inhibitory activity from Pogonatherum crinitum. J Ethnopharmacol. 2008:118:71-8.

32. Lu CL, Zhu W, Wang DM, Chen WL, Hu MM, Wang M, et al. Inhibitory effects of chemical compounds isolated from the rhizome of Smilax glabra on nitric oxide and tumor necrosis factor-a production in lipopolysaccharide-induced RAW264.7 cell. Evid-Based Compl Alt. 2015;2015:602425

33. Park HY, Kim GY, Choi YH. Naringenin attenuates the release of pro-inflammatory mediators from lipopolysaccharide-stimulated BV2 microglia by inactivating nuclear factor- $\mathrm{KB}$ and inhibiting mitogen-activated protein kinases. Int J Mol Med. 2012;30:204-10.

\section{Publisher's Note}

Springer Nature remains neutral with regard to jurisdictional claims in published maps and institutional affiliations.

Ready to submit your research? Choose BMC and benefit from

- fast, convenient online submission

- thorough peer review by experienced researchers in your field

- rapid publication on acceptance

- support for research data, including large and complex data types

- gold Open Access which fosters wider collaboration and increased citations

- maximum visibility for your research: over 100M website views per year

At BMC, research is always in progress.

Learn more biomedcentral.com/submissions 